\title{
43. Übersetzungsrelevante Textanalyse und Übersetzungsprobleme am Beispiel eines Kinderbuches: Die Konferenz der Tiere von Erich Kästner
}

\section{Ümmügülsüm ALBİZ}

\begin{abstract}
APA: Albiz, Ü. (2021). Übersetzungsrelevante Textanalyse und Übersetzungsprobleme am Beispiel eines Kinderbuches: Die Konferenz der Tiere von Erich Kästner. RumeliDE Dil ve Edebiyat Araşttrmaları Dergisi, (23), 685-707. DOI: 10.29000/rumelide.949652.
\end{abstract}

\section{Zusammenfassung}

In dieser vorliegenden Arbeit werden ausgehend von dem Werk von Christiana Nords " Textanalyse und Übersetzen, Theoretische Grundlagen, Methode und Didaktische Anwendung einer übersetzungsrelevanten Textanalyse", übersetzungsrelevante Textanalyse und Übersetzungsprobleme des Werkes "Die Konferenz der Tiere" von Erich Kästner thematisiert. Mit dieser Arbeit werden zuerst die Textfunktion (Texttypen und Textsorten), später Übersetzungstypen und Übersetzungsmodelle von Nord aufgeklärt, die der Laswell-Formell zugründe gelegen wurden. Das Hauptziel dieser Arbeit ist es, die Bedeutung dieser textexternen und textinernen Faktoren vor dem Übersetzungsprozess hervorzuheben und einen Bewusstsein für eine funktionale Textanalyse $\mathrm{zu}$ entwickeln. Mit diesen Faktoren werden pragmatische, konventionsbezogene, sprachenpaarbezogene und textspezifische Übersetzungsprobleme am Beispiel eines Kinderbuches diskutiert. Durch dieses Kinderbuch werden textexterne und textinterne Faktoren sowohl des Ausgangstextes als auch des Zieltextes und mögliche Übersetzungsprobleme nach Nords Ansatz bestimmt. Ausgehend von diesem Ansatz wird bestimmt, dass pragmatische, konventionsbezogene, besonders sprachenpaarbezogene Übersetzungsprobleme in dem Zieltext entstehen und die berufserfahrene Übersetzerin, Süheyla Kaya, diese Probleme mit ihrer Kultur- und Sprachkompetenz überwindet. Da dieses Werk literarisches Buch bzw. ein Kinderbuch ist, ist identisch die Funktion des Ausgangstextes mit der Funktion des Zieltextes.

Schlüsselwörter: Übersetzungswissenschaft, übersetzungsrelevante Textanalyse, textexterne und textinterne Faktoren, Übersetzungsprobleme, die Konferenz der Tiere von Erich Kästner

\section{Bir çocuk kitabı örneğinde çeviri amaçlı metin analizi ve çeviri problemleri: Erich Kastner'in Hayvanlar Toplantssı}

\section{$\ddot{O} \mathbf{z}$}

Mevcut çalışmada, Christiana Nord'un "Metin Analizi ve Çeviri, Çeviri Amaçlı Metin Analizinin Teorik Temelleri, Metotları ve Didaktik Uygulaması" adlı yapıtından hareketle Erich Kästner'in "Hayvanlar Konferansı" adlı eseri, çeviri amaçlı metin analizi ve çeviri problemleri açısından konu edinilmektedir. Bu çalışma ile öncelikle metnin işlevi (metin tipleri ve türleri) daha sonra ise Nordun çeviri türleri ve Laswell- Formülüne dayandırılmış olan çeviri modeli açıklanmaktadır. Çalışmanın asıl amacı, çeviri sürecinden önce metin içi ve dışı faktörleri ortaya koymak ve işlevsel metin analizi için bir bilinç geliştirmektir. Bu faktörlerin yanısıra, pragmatik, gelenek, dil çifti ve metin türü ile ilgili çeviri problemleri, çocuk kitabı örneğinde tartışılmaktadır. Bu çocuk kitabı

Dr. Öğr. Üyesi, Karamanoğlu Mehmet Bey Üniversitesi, Yabancı Diller Yüksekokulu, Mütercim Tercümanlı Bölümü, Almanca Mütercim Tercümanlı ABD (Karaman, Türkiye), ualbiz@kmu.edu.tr, ORCID ID: oooo-0002-2231-8672 [Araştırma makalesi, Makale kayıt tarihi: 26.04.2021-kabul tarihi: 20.06.2021; DOI: 10.2900o/rumelide.949652]

Adres | Address

RumeliDE Dil ve Edebiyat Araşttrmaları Dergisi $\quad$ RumeliDE Journal of Language and Literature Studies Osmanağa Mahallesi, Mürver Çiçeği Sokak, No:14/8 Osmanağa Mahallesi, Mürver Çiçeği Sokak, No:14/8

Kadıköy - İSTANBUL / TÜRKIYE 34714 Kadıköy - ISTANBUL / TURKEY 34714 e-posta: editor@rumelide.com

e-mail: editor@rumelide.com

tel: +90 505 7958124, +90 2167730616 phone: +90 505 7958124, +90 2167730616 
Text analysis for translation and translation problems in a children's book example:Erich Kästner's Animal Meeting / Ü. Albiz (pp. 685-707)

sayesinde, hem kaynak hem de erek metnin metin içi ve dışı faktörleri ve olası çeviri problemleri, Nord'un yaklaşımına göre tespit edilmektedir. Bu yaklaşımdan hareketle erek metinde pragmatik, geleneksel, özellikle dil çifti bazındaki çeviri problemlerinin ortaya çıttı̆̆ı ve deneyimli çevirmen Süheyla Kaya'nın, bu problemleri dil ve kültür edinci ile aşttğı tespit edilmektedir. Bu eser, edebi bir kitap, bir çocuk kitabı olduğu için, kaynak metnin işlevi, erek metnin işlevi ile aynı özellikleri taşımaktadır.

Anahtar kelimeler: Çeviribilim, çeviri amaçlı metin analizi, metin içi ve dışı faktörler, çeviri problemleri, Erich Kästner’in Hayvanlar Toplantısı

\title{
Text analysis for translation and translation problems in a children's book example:Erich Kästner's Animal Meeting
}

\begin{abstract}
In this study Erich Kästner's “Die Konferenz der Tiere (Animal Meeting)” in terms of text analysis for translation purposes and translation problems is dwelt upon in relation to Christiana Nord's "Textanalyse und Übersetzen, Theoretische Grundlagen, Methode und Didaktische Anwendung einer Übersetzgunsrelevanten Textanalyse". With the study, firstly the function of the text (text types), and subsequently, Nord's text types and translation model based on Laswell- formula are explained. The main aim of the study is to reveal in-text and out-of-text factors before the translation process and to develop a consciousness for functional text analysis. Apart from these factors, translation problems related to pragmatic, tradition, language pair and text type are discussed in the example of a children's book. By means of this children's book, in-text and out-oftext factors of both source and target text are detected in accordance with Nord's approach. Within the frame of this approach, it is observed that pragmatic, traditional, especially language pair-based translation problems arise in the target text and experienced translator Süheyla Kaya overcame these problems with language and cultural competence. Since this work is a literary book, a children's book, the function of source text has the same features with the function of the target text.
\end{abstract}

Keywords: Translation studies, text analysis for translation purposes, factors in and out of text, translation problems, Erich Kästner's Die Konferenz der Tiere (Animal Meeting)

\section{Einführung}

Diese Arbeit bezweckt nach Christiana Nord einen Ausgangstext und Zieltext im Hinblick auf textexterne und textinterne Faktoren zu analysieren. Aber aushegend von Nords Ansätzen sollen zuerst Textfunktion und Übersetzungstypen aufgeklärt werden. Danach werden kurz theoretische Voraussetzungen, Aufgaben und Faktoren der Ausgangstextanalye unter dem Titel der übersetzungsrelevanten Ausgangstextanalyse in dieser Reihenfolge auseinandergesetzt und schließlich wird dieses Modell am Beispiel eines Kinderbuches untersucht.

Seit den 1970er Jahren und besonders in der deutschen Übersetzungswissenschaft (Reiss, Thiel, Wills, Koller usw.) wird die Wichtigkeit der übersetzungsrelevanten Textanalyse zum Thema gemacht, aber Nord behauptet, dass Textanalysen übersetzerische Entscheidungen erleichtern und auf alle Textsorten anwendbar sind (Cseho, 2007: 63). Nord (2009:2)berichtet, dass ein solches Modell unabhängig von den Spezifika der Ausgangs- oder Zielsprache, unabhängig von der Adres $\mid$ Address

RumeliDE Dil ve Edebiyat Araştırmaları Dergisi $\quad$ RumeliDE Journal of Language and Literature Studies Osmanağa Mahallesi, Mürver Çiçeği Sokak, No:14/8 Osmanağa Mahallesi, Mürver Çiçeği Sokak, No:14/8 Kadıköy - İSTANBUL / TÜRKIYE 34714 Kadıköy - ISTANBUL / TURKEY 34714 e-posta: editor@rumelide.com

e-mail: editor@rumelide.com

tel: +90 505 7958124, +90 2167730616 phone: +90 505 7958124, +90 2167730616 
Übersetzungsrichtung und unabhängig auch vom Kompetenzgrad des Übersetzers erdacht werden muss.

\section{Text und textfunktion}

Nord (1993:8) behauptet, dass die funktionale Neuorientierung der Übersetzungswissenschaft an den Erfordernissen der professionellen Praxis ausgerichtet ist und erfordert, das Übersetzen von Gebrauchstexten und von literarischen Texten unter einen theoretisch-methodischen Hut zu bringen. Diese funktionale Neuorientierung basiert auf der Skopostheorie von Reiss/ Vermeer, die den Zweck einer Translationshandlung und kommunikative Funktion des Textes hervorbringen. Deshalb ist es wichtig, zu bestimmen, welche Funktionen ein Text bezweckt, denn ein Übersetzer/eine Übersetzerin soll nicht beginnen zu übersetzen, bevor er die Textfunktion festlegt. Im Grunde genommen bezweckt man, eine Übersetzung zwischen zwei Sprachen und Kulturen eine kommunikative Funktion zu verfügen. Unter dem Begriff der Textfunktion versteht man die kommunikative Funktion bzw. die Kombination aus den kommunikativen Funktionen eines Textes in seiner konkreten (Rezeptions)Situation aber ein Text hat also nicht eine Funktion, sondern der Text erhält eine Funktion in der Rezeptionssituation (Nord, 1993: 9). Textexterne Faktoren wie Sender, Empfänger, Ort, Zeit, Medium usw. spielen wichtige Rolle in der Rezeptionssituation.

Nord (1993: 10) stützt sich auf eine Synthese aus den Funktionsmodellen von Bühler und Jakobson und laut Nord sind vier Grundfunktionen der Kommunikation auseinanderzuhalten.

1. Mitteilung über die Gegenstände und Erscheinungen der außersprachlichen Welt (=referentielle oder Darstellungsfunktion)

2. Ausdruck der Einstellung des Senders zu den Gegenständen und Erscheinungen der Welt (=expressive oder Appelfunktion)

3. Appell an den Empfänger (=operative oder Appellfunktion)

4. Herstellung, Aufrechterhaltung und Beendigung von Kontakt (=phatische Funktion).

Nord (1993: 11) betont, dass die Funktion des Textes (auf der Empfängerseite) der Intention (auf der Senderseite) entspricht. Deshalb ist es wichtig für Übersetzer, die Funktion des Textes zu bestimmen. Der Übersetzer schafft das, wenn er die Textklassifizierung macht. Und die Textklassifizierung des Übersetzers führt den Übersetzer im Übersetzungsprozess und ermöglicht bewusste und wahrhaftige Übersetzungsentscheidungen. Kautz (2002: 55) unterstreicht auch, dass die Funktion eines Textes mit der Intention seines Verfassers zusammenhängt, aber sie wird auf den Text wegen seines Adressaten verweist. Er hebt hervor, dass die Funktion des Ausgangstextes mit der Funktion des Zieltextes identisch sein kann.

Während der Ausgangstextanalyse, die die Funktion und den Zweck unterscheidet, stellt Nord infrage, wer den Translationszweck bestimmt. Als Antwort auf diese Frage gibt sie "Translationssituation", die nicht von eigenem Antrieb des Übersetzers / der Übersetzerin, sondern von dem Auftrag des Kunden oder Auftraggebers ${ }^{2}$ bestimmt wird (Nord, 1993: 14-15). Weil die Normen oder Konventionen für die Rezeption von Übersetzungen noch nicht im Detail erforscht sind, hat Nord neben das Kriterium der Funktionsgerechtigkeit, das der Loyalität gestellt. Nord (2006: 33) bringt zum Ausdruck, dass die Verantwortung des Translators gegen seine Partner Loyalität ist. Das Loyalitätsprinzip findet erst mal in der Skopostheorie 1989 statt.

2 Nord nennt den Auftraggeber als Initiator.

RumeliDE Dil ve Edebiyat Araşttrmalar Dergisi Osmanağa Mahallesi, Mürver Ciçeği Sokak, No:14/8 Kadıköy - ISTANBUL / TÜRKIYE 34714 e-posta: editor@rumelide.com tel: +90 505 7958124, +90 2167730616
Address

RumeliDE Journal of Language and Literature Studies

Osmanağa Mahallesi, Mürver Çiçeği Sokak, No:14/8

Kadıköy - ISTANBUL / TURKEY 34714

e-mail: editor@rumelide.com,

phone: +90 505 7958124, +90 2167730616 
Text analysis for translation and translation problems in a children's book example:Erich Kästner's Animal Meeting / Ü. Albiz (pp. 685-707)

Da es nun der Translator in den Rollen der Autoren, der Initiatoren/ Auftraggeber und der Zieltextrezipienten mit Personen zu tun hat, die sein Vorgehen kaum oder überhaupt nicht überprüfen können, darf er seine Partner nicht hintergehen, deshalb ist er ihnen gegenüber zur Loyalität verpflichtet (Prunc, 2002:192).

Durch die Loyalität versteht man, dass Übersetzer oder Übersetzerinnen gegenüber ihren Handlungspartnern, den Auftraggebern, Zieltextempfängern und dem Autor/der Autorin des Ausgangstextes in der Verantwortung stehen (Nord, 1993: 18). Der Übersetzer/die Übersetzerin übernimmt die Verantwortung der Loyalität gegenüber dem Zieltextempfänger und dem Ausgangsverfasser (Stolze,2005:191). Wegen dieser Verantwortung soll der Übersetzer/die Übersetzerin nicht bewusst falsche Abweichungen von Übersetzungen verwirklichen, aber er/sie muss die kulturspezifische Konventionen oder Normen ins Auge fassen.

\section{1. Übersetzungstypen: dokumentarisches vs. instrumentelles übersetzen}

Unter diesem Titel behandelt man kurz die von Nord verwendeten funktionale ÜbersetzungstypenDokumantarische Übersetzung und Instrumentelle Übersetzung- mit ihren Unterfunktionen. Zuerst soll man die Details der dokumantarichen und Instrumentalen Übersetzung erklären.

Eine Übersetzung kann in zwei Funktionsrelationen als Dokument und Instrument zum Ausgangstext beobachtet werden. Während Dokument die ausgangsprachliche Kommunikationshandlung für den Zieladressaten abbildet, ist Instrument hinsichtlich der bestimmten Merkmale am Vorbild des ausgangssprachlichen Kommunikatiosinstrument orientiert. In dem zweiten Fall geht es um die Herstellung der Kommunikation zwischen dem Ausgangstextsender und dem Zieltextempfänger (Nord, 1993: 24-25).

Während die dokumentarische Übersetzung als Dokument der Situation fungiert, in welcher der Verfasser des Ausgangstextes mit seinem Zielpublikum kommuniziert hat, dient eine instrumentelle Übersetzung als Instrument zur Erreichung eines kommunikativen Zieles in einer neuen, zielkulturellen Kommunikationshandlung (Prunc, 2002: 188). Nord betont, dass jeder Text -also Ausgangs- oder Zieltext- eine referentielle, expressive, appellative oder phatische Funktion hat, die auf die Situation Bezug nimmt. Weil Rezeptionssituationen der Zieltextadressaten sich ändern, kann sich die Funktion des Textes auch ändern.

Dokumentarische Übersetzung hat vier Übersetzungstypen als Wort für Wort Übersetzung, wörtliche Übersetzung, philologische und exotisierende Übersetzung, die in dem Zieltext metatextuelle Funktion haben. Instrumentelle Übersetzung ist auch drei Übersetzungstypen- funktionskonstante, funktionsvariierende und korrespondierende/homologe Übersetzung-, die in dem Zieltext referentielle oder phatische Funktionen haben. Um die Translationstypologie von Nord ausführlich aufzufassen, soll man dokumentarische und instrumentelle Übersetzung mit ihren jeweiligen Übersetzungstypen betrachten.

\section{2. Übersetzungsrelevante textanalyse und übersetzen}

Nord gründet ihr Modell auf die Übersetzungsansätze von Reiss und Vermeer und Textanalyseschema. Ausgehend von einem funktionalen Translationsverständnis bezweckt Nord eine modellhafte Darstellung des Translationsvorgangs. Nach Meinung von Nord handelt es sich um einen Unterschied zwischen Translationsvorgang und Translationsprozess. Während der Translationsprozess ein Teil des

\footnotetext{
Adres | Address

RumeliDE Dil ve Edebiyat Araşttrmaları Dergisi $\quad$ RumeliDE Journal of Language and Literature Studies Osmanağa Mahallesi, Mürver Çiçeği Sokak, No:14/8 Osmanağa Mahallesi, Mürver Çiçeği Sokak, No:14/8 Kadıköy - İSTANBUL / TÜRKIYE 34714 Kadıköy - ISTANBUL / TURKEY 34714 e-posta: editor@rumelide.com e-mail: editor@rumelide.com, tel: +90 505 7958124, +90 2167730616 phone: +90 505 7958124, +90 2167730616
} 
Translationsvorgangs ist, hat der Translationsvorgang translatorunabhägige Faktoren. Und Nord erklärt den Translationsvorgang ausführlich so;

Der Translationsvorgang wird in der Regel dadurch in Gang gesetzt, dass sich ein Auftraggeber, den ich Initiator (I) nennen will, an einen Translator (TRL) wendet, weil er einen bestimmten Zieltext (ZT) für einen Rezipienten (ZT-R) benötigt bzw. weil er einen in einer Ausgangssprache (AS) und unter bestimmten ausgangskulturellen Bedingungen von einem Textproduzenten (AT-P) verfassten und von einem Textsender (AT-S) gesendeten Text (AT) selbst in der Zielsprache rezipieren will (Nord,2009: 5).

Erläuternd, wie der Translationsvorgang beginnt, berichtet Nord, dass eine Unterscheidung vom Ausgangstextsender (AT-S) und Ausgangstextproduzent (AT-P) notwendig und der Translator der reale Empfänger des Ausgangstextes im Translationsvorgang ist. Bedienungsfaktoren und Konstituenten des Translationsvorgangs sind nach der Reihe Ausgangstextproduzent, Ausgangstextsender, Ausgangstext, Ausgangstextrezipient, Initiator, Translator, Zieltext und Zieltextrezipient. Ausserdem hebt Nord hervor, dass der Translator bei der Textanalyse den Situationsfaktoren der Ausgangssituation Rechnung tragen muss, weil Nord Aufmerksamkeit auf die Situation ziehen will.

Während Nord von der Rolle des Initiators erwähnt, impliziert sie, dass der Initiator den Translationsvorgang beginnen lässt, weil er einen bestimmten Zieltext, nämlich ein "Translat" braucht. Nord bezeichnet den Initiator als entscheidender Faktor für den Translationsskopos. Wenn Nord von der Rolle des Translators handelt, der in ihrem Schema des Translationsvorgangs eine zentrale Position hat, stellt sie den Translator sowohl Rezipient des Ausgangstextes und als auch Produzent des Zieltextes dar. Aber der Translator rezipiert nicht den Ausgangstext für seinen eigenen Zweck, sondern er rezipiert den AT anstelle des Initiators bzw. des vom Initiator bestimmten ZT-Rezipienten (Nord, 2009: 9-11).

Um texttheoretische Grundlagen zu erläutern, determiniert Nord drei wichtigsten Titeln "Zum Textals Handlungsbegriff, Rezeptionsbedingungen und Textsorten und Texttypen”. Ein Text kann nur im Rahmen der Handlung-in- Situation analysiert werden, in welcher der Text mit seinen Funktionen seinen Platz hat, und erfüllt den intendierten Zweck durch die kommunikative Funktion. Die kommunikative Funktion lässt sich nicht nur vom Sender als auch vom Empfänger analysieren, falls der Text als kommukivativer Signalverbund in der Kommunikationssituation zu betrachten ist (Nord, 2009: 12-16). Der Text als Zeichen der kommunikativen Funktion wird von der Intention des Senders, Textproduzenten oder Empfängers gestaltet. Rezeptionsbedingungen verändern sich nach Intentionen, Erwartungen und Situationen. Mehrere Textsorten werden meist auf einer weiteren Abstraktionsebene zu Texttypen (Reiss oder Werlich) oder Textklassen (z.B. bei Grosse und Scmidt) oder Textsortenklassen zusammengefasst, wobei unterschiedliche Klassifizierungsmassstäbe als Grundlage dienen (Nord, 2009: 22). Für den Translator ist es wichtig, übersetzungsrelevante Texttypologie zu bestimmen. Nach Reiss besteht die übersetzungsrelevante Texttypologie aus informativen, expressiven, operativen und audio-medialen Texten. Während das Kennzeichen des informativen Textes seine Sachorientiertheit ist, ist das Kennzeichen des expressiven Texttyps seine Senderorientiertheit und das Kennzeichen des operativen Textes ist seine Verhaltensorientiertheit; für den audio-medialen Text trifft je nach Textsorte grundsätzlich auch eine dieser drei Übersetzungsmethoden zu (Reiss, 1993: 20-23). Textklassifizierung oder Textklassifizierungsansätze führen den Translator zu der übersetzungsrelevanten Textanalyse, dadurch findet der Translator seinen Weg, um eine funktionale Übersetzung und eine zielorientierte Übersetzung durchzuführen.

\footnotetext{
Adres
RumeliDE Dil ve Edebiyat Araștırmaları Dergisi Osmanağa Mahallesi, Mürver Ciçeği Sokak, No:14/8 Kadıköy - ISTANBUL / TÜRKIYE 34714 e-posta: editor@rumelide.com tel: +90 $5057958124,+902167730616$ 
Text analysis for translation and translation problems in a children's book example:Erich Kästner's Animal Meeting / Ü. Albiz (pp. 685-707)

Um Aufgaben der Ausgangstextanalyse festzulegen, betont Nord, dass die Relationen zwischen Ausgangs und Zieltext auf Treue, Freiheit und Äquivalenz bassiert. Und sie deutet an, dass funktionale Äquivalenz zwischen Ausgangstext und Zieltext ein Sonderfall ist, bei dem der Faktor Funktionswechsel "zero" gesetzt wird (Nord, 2009: 25). Erwähnend von der Skopostheorie und dem translatorischen Handeln im Rahmen der theoretischen Ansätzen entwickelt Nord einen theoretischen Hintergrund für ihre Ansichten, d.h. übersetzungsrelevante Textanalyse.

\begin{abstract}
Ausgangspunkt für eine Translation ist auch in dieser Theorie ein Text der Sprache A, der in eine Sprache $Z$ übertragen und dann ein für Z-Empfänger als "kohärent mit ihrer Situation" interpretierbarer Teil eines Weltkontinuums werden soll. Das Verhältnis zwischen Ziel- und Ausgangstext soll ebenfalls mit dem Begriff "Kohärenz" (intertextuelle Kohärenz: Fidelität) beschreibbar sein (Nord, 2009: 26).
\end{abstract}

Alle Elemente des Soll-Zustandes des Zieltextes im Ist-Zustand des Ausgangstextes vorzugeben und Informationslücken des Translators auszugleichen, sind sehr wichtig für die Kompatibilität der ZTFunktion mit dem AT. Und ausgehend von translatorischem Handeln betont Nord, dass ein ZT nicht als Translation sondern als translatorisches Handeln bezeichnet wird, falls jeweiligen Zieltext ohne Anbindung an einen Ausgangstext produziert wird. Nord bezeichnet auch die Verantwortung des Translators gegenüber dem AT-Sender, Auftraggeber und ZT-Empfänger als Loyalität, die eine ethische Kategorie im Zusammenleben von Menschen ist. Um die Phase des Translationsprozesses neben Zwei-Schritt-Schema und Drei-Schritt-Schema weiterzuentwickeln, setzt Nord ein Zirkelschema vor, das zwischen AS-Situation und AT bzw. ZS-Situation und ZT, zwischen den einzelnen Analyseschritten und zwischen AT-Analyse und ZT-Synthese rekursive Kreisbewegungen im Kleinen enthält.

\title{
2.1. Faktoren der ausgangstextanalyse
}

Reiss, Bühler, Hönig und Nord haben die Laswell-Formell gewählt, mit deren Abfrageschema sie einen Ausgangspunkt für ihre übersetzungsrelevante Textanalyse betätigt haben. Nord (1999: 351) hebt die Fassung des Abfrageschemas ausführlich hervor; "Wer übermittelt wozu wem über welches Medium wo wann warum einen Text mit welcher Funktion? Worüber sagt er was (was nicht) in welcher Reihenfolge, unter Einsatz welcher nonverbalen Elemente, in welchen Wörten, in was für Sätzen, in welchem Ton mit welcher Wirkung? Ausgehend von dieser genannten Abfrageschemata bestimmt Nord textexterne, textinterne Faktoren der Ausgangstextanalyse und Wirkung und legt die didaktische Anwendung des Modells dar.

Nord (2009: 40) ordnet textexterne und textinterne Faktoren des Ausgangstextes durch W-Fragen wie folgenden zu;

wer übermittelt

wem

wozu

über welches Medium

wo

wann

warum einen Text

mit welcher Funktion? worüber sagt er/sie

was

(was nicht)

in welcher Reihenfolge

unter Einsatz welcher nonverbalen Elemente

in welchen Worten

in was für Sätzen

in welchem Ton

mit welcher Wirkung?

RumeliDE Dil ve Edebiyat Araşttrmaları Dergisi Osmanağa Mahallesi, Mürver Cicceği Sokak, No:14/8 Kadıköy - İSTANBUL / TÜRKIYE 34714 e-posta: editor@rumelide.com tel: +90 $5057958124,+90216773$ o 616
Address

RumeliDE Journal of Language and Literature Studies Osmanağa Mahallesi, Mürver Çiçeği Sokak, No:14/8

Kadıköy - ISTANBUL / TURKEY 34714

e-mail: editor@rumelide.com

phone: +90 $5057958124,+90216773$ o 616 


\subsubsection{Textexterne faktoren}

Textexterne Faktoren werden durch diese W-Fragen erfasst; Wer (Textproduzent bzw. Sender), Wozu (Senderintention), Wem (Adressat), über welches Medium (Medium bzw. Kanal) und Wo (Ort), Wann (Zeit), Warum (Anlass) und schliesslich mit welcher Funktion?

In dem Modell von Nord werden Sender und Senderintention als getrennte Faktoren betrachtet, während diese Begriffe in den meisten Ansätzen nur als ein Faktor behandelt werden. Denn Nord hebt hervor, dass es sich um die unterschiedlichen Einflüsse von diesen zwei Begriffen auf den textinternen Faktoren handelt. Obwohl ein oder dieselbe Person, Sender und Verfasser eines Textes (bei literarischen, sachlichen Texte oder Zeitungskommentar, -leitartikeln) ist, betont Nord, dass die Unterscheidung für die übersetzungsrelevante Textanalyse eine grosse Rolle spielt, und viele Textsorten vor allem Gebrauchsanweisungen wie medizinische Packungsbeilage, Reklametexte, Gesetzestexte keinen bestimmten Verfasser haben. Aber in jedem Fall ist ein Sender vorhanden. Und Senderintention kann durch manche Leitfragen ermittelt werden, um zu bestimmen, ob es textexterne explizite Äusserungen des Senders zu seiner Intention gibt oder welche Intentionen mit der Textsorte konventionelle verbunden sind, usw. Im Hinblick auf Empfängerpragmatik müssen Empfängerdaten wie Alter, Geschlecht, Bildungsstand, gesellschaftliches Umfeld, geographische Herkunft betrachtet werden. "Über welches Medium" ist noch eine andere gestellte Frage, die den Faktor Medium/Kanal analysieren muss und medienspezifische Merkmale im Inhalt und in der Form des Textes festlegen muss. Eine andersartige Frage ist auch: Wo und wann wurde der Text gesendet? Mit dieser Frage können die Informationen zur Ortspragmatik und Zeitpragmatik des Textes entommen werden. "Warum" ist die andere Frage in der pragmatischen W-Kette, um den Anlass des Textes determinieren zu können.

\subsubsection{Textinterne faktoren}

Um die textexternen Faktoren zu bestimmen, sind gestellte Fragen wie unten:

Worüber (Thematik), Was (Textinhalt), Was nicht (Präsuppositionen), in welcher Reihenfolge (Textaufbau), in welchen Worten (Lexik), in was für Sätzen (Syntax) und in welchem Ton (suprasegmentale Merkmale)

Die Textthematik ist von Bedeutung in den Ansätzen von Wills, Cartellieri, Thiel und Reiss. Sie bestimmen die Rolle zur übersetzungsrelevanten Textanalyse. Unter Textinhalt werden die Titeln wie die Paraphrase als Verfahren, Kohäsionsmerkmale des Textes, Konnotation und Sekundärinhalt, zur inneren Situation behandelt.

\subsubsection{Wirkung}

Das Zusammenspiel der textexternen und internen Faktoren wird durch die Wirkung dargelegt. Und Nord erzählt von den einigen Faktorenrelationen wie Senderintention-Text; Empfänger-Textwelt; Empfänger-Stil und von den Wirkungstypen wie Intentionsgemässe vs. nicht-intentionsgemässe Wirkung; Kulturelle Distanz vs. Zero-Distanz; Konventionalität vs. Originalität.

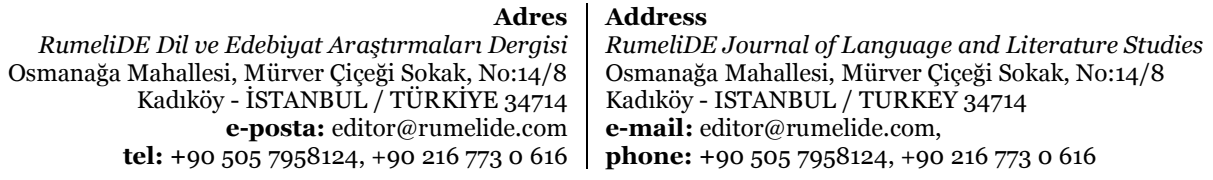

tel: +90 505 7958124, +90 2167730616 
Text analysis for translation and translation problems in a children's book example:Erich Kästner's Animal Meeting / Ü. Albiz (pp. 685-707)

\section{2. Übersetzungsprobleme}

Nord behandelt die Übersetzungsprobleme in vier Kategorien, die "top-down" nämlich von der Situation zu den sprachlichen Merkmalen angeordnet sind.

\section{Pragmatische Übersetzungsprobleme (PÜP)}

Wenn ein Ausgangstext in eine andere Zielsprache oder verschiedene Zielsprache übersetz wird, können konkrete Übersetzungsprobleme aus der Übersetzungssituation ableiten. Nord stellt klar, dass das Gegenteil von Ausgangs- und Zielempfänger, Ausgangs- und Zielmedium, AusgangstextProduktionsanlass und Translationsanlass $\mathrm{zu}$ den Übersetzungsprobleme Anlass gibt. Und pragmatische Übersetzungsprobleme sind meistens in Bezug auf die textexternen Faktoren wie Sender-,Zeit-, Orts-, Empfängerpragmatik usw. (Nord, 2009:177).

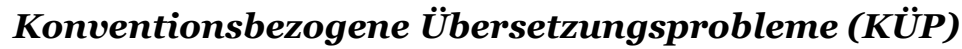

Konventionsbezogene Übersetzungsprobleme werden in der dritten Auflage3 des Werkes "Textanalyse und Übersetzen" von Nord "kulturpaarspezifische Übersetzungsprobleme genannt, aber weil manche Missverständnisse darüber zustande kommen, nennt Nord sie in der vierten überarbeiteten Auflage4 "konventionsbezogene Übersetzungsprobleme". Die Konvention, die aus der Kultur, Tradition, Gewohnheit besteht, macht Verhaltensweise durchschaubar, die durch Normen und Konventionen gelenkt werden.

\section{Sprachenpaarbezogene Übersetzungsprobleme (SÜP)}

Sprachenpaarbezogene Übersetzungsprobleme sind unvermeidlich, weil es sich um strukturelle Unterschiede insbesondere hinsichtlich der Lexik und Syntax zwischen jede zwei Sprachen handelt. Nord unterstreicht die Differenze zwischen Problemen der Sprachbeherrschung und der Übersetzungskompetenz.

\section{Text(exemplar)spezifische Übersetzungsprobleme (TüP)}

Laut Nord ist textspezifisches Übersetzungsproblem ein Sonderfall, dessen Lösung nicht verallgemeinert werden kann. Anhand der verschiedenen Texttypen wirft man ein Licht, in welchen Textsorten oder bei welchen Übersetzungsaufträgen bestimmte Problemtypen auftreten (Nord, 2009: 179).

\section{Anwendung des modells der übersetzungsrelevanten textanalyse: am bespiel von Erich Kästners werk "Die Konferenz der Tiere"}

In dieser Arbeit werden nach dem "Top-down" Verfahren von Nord zuerst die textexternen und dann textinternen Faktoren zu analysieren.

\footnotetext{
3 Textanalyse und Übersetzen, Theoretische Grundlagen, Methode und didaktische Anwendung einer übersetzungsrelevanten Textanalyse, 3. Auflage, Julius Groos Verlag Heidelberg, s.182.

4 Textanalyse und Übersetzen, Theoretische Grundlagen, Methode und didaktische Anwendung einer übersetzungsrelevanten Textanalyse,4., überarbeitete Auflage, Julius Groos Verlag Tübingen.

Adres | Address

RumeliDE Dil ve Edebiyat Araşttrmaları Dergisi $\quad$ RumeliDE Journal of Language and Literature Studies Osmanağa Mahallesi, Mürver Çiçeği Sokak, No:14/8 Osmanağa Mahallesi, Mürver Çiçeği Sokak, No:14/8

Kadıköy - İSTANBUL / TÜRKIYE 34714 Kadıköy - ISTANBUL / TURKEY 34714 e-posta: editor@rumelide.com e-mail: editor@rumelide.com,

tel: +90 505 7958124, +90 2167730616 phone: +90 505 7958124, +902167730616
} 


\subsection{Textexterne faktoren des ausgangstextes}

\section{Sender}

Ausgehend von Quellenangaben, Klappen- oder Umschlagstexten, Vor- oder Nachworten oder Fussnoten usw. ermittelt man die Informationen des Senders. Während der Verfassername Informationen über die Weltwissen des Rezipienten oder Translators gibt, bedeutet Nord, dass der Translator durch Zusatzinformationen kompensieren muss, wenn ein Informationsdefizit für die Zieltextrezipienten besteht.

Der Sender des Ausgangstextes ist Erich Kästner, wer 1899 in Dresden geboren wurde und 1974 in München starb. Er ist ein berühmter Autor mit seinen Kinderwerken wie "Das doppelte Lottchen", "Pünktchen und Anton", "Das fliegende Klassenzimmer" und "Emil und Detektive". Der berühmte Autor wurde durch diesen Werken mit zahlreichen Preisen bedacht. Er ist überzeugt, dass die Erde besser sein könnte, wenn die Leute vernünftiger wären und Kinder sind ihm Garanten der Möglichkeit, die humana Substanz zu retten (Kutlu,2006: 14)

\section{Senderintenion}

Um Senderintention festzulegen, ist die im Vordergrund stehende Frage: Was will der Sender mit dem Text (beim Empfänger) bewirken? Ausserdem ist die Intention wegen der Loyalität wichtig, denn der Translator darf nie die Intention des Senders verändern.

Ausgehend von der obengegebenen Frage können wir sagen, dass der Autor als Sender mit diesem Kinderbuch, das nach dem zweiten Weltkrieg geschrieben wurde, bezweckt, Weltfrieden und Hoffnung mit den Vertretern aller Tierarten auf der Welt zu erreichen, bzw. um ein gewaltfreies Zusammenleben für Empfänger zu schaffen.

\section{Empfängerpragmatik}

Nord betont, dass eine Übersetzung sich niemals an dieselben Empfänger wie das Original richtet, weil die Empfänger des Zieltextes in einer anderen Kultur und Gesellschaft leben. Daher bringt Nord zum Ausdruck, dass die Überlegungen zur Empfängerpragmatik nicht nur auf die Ausgangstextrezipienten oder -adressaten und ihr Verhältnis zum Ausgangstextsender sondern auch auf die Zieltextadressaten richten, deren Erwartungen, Vorkenntnisse und Stellung im Kommunikationsvorgang das Vertextungsverhalten des Zieltextproduzenten, also des Translators, bestimmen (Nord, 2009: 60).

Im Rahmen dieser Werksanalyse kann man behaupten, dass der Empfängerkreis dieses Buches aus den 8-10 jährigen deutschen Kindern besteht. Es handelt sich um keine bestimmte Kennzeichnung mit der Adressatengruppe in diesem Buch, weil es kein Vorwort und Schluswort hat. Aber am Ende des Buches wird dieses Werk als ein Kinderbuch unter dem Titel "Kinderbücher von Erich Kästner" bestätigt. Durch Recherche über dieses Buch werden Kenntnisse mit Lesealter5 leicht erworben.

5 Umfangreiche Informationen über den Verlag https://www.amazon.de/Die-Konferenz-Tiere-Erich $\mathrm{K} \% \mathrm{C}_{3} \%$ A4stner/dp/3791530216

RumeliDE Dil ve Edebiyat Arasturmatari Address

RumeliDE Journal of Language and Literature Studies Osmanağa Mahallesi, Mürver Çiçeği Sokak, No:14/8 Osmană̆a Mahallesi, Mürver Çiçeği Sokak, No:14/8

Kadıköy - ISTANBUL / TÜRKIYE 34714 Kadıköy - ISTANBUL / TURKEY 34714 e-posta: editor@rumelide.com e-mail: editor@rumelide.com,

tel: +90 505 7958124, +90 2167730616 phone: +90 505 7958124, +90 2167730616 
Text analysis for translation and translation problems in a children's book example:Erich Kästner's Animal Meeting / Ü. Albiz (pp. 685-707)

\section{Medium/Kanal, Ortspragmatik, Zeitpragmatik}

Unter diesem Titel begreift man das Transportmittel oder -vehikel, mit dessen Hilfe der Text an die Empfänger gelangt und die mündliche oder schriftliche Kommunikationssituation des Textes wirkt sowohl auf die Bedingungen der Rezeption als auch auf die Produktion aus. Weil die Medienlandschaft von dem historsichen Wandel und kulturspezifischen Unterschieden betroffen sind, werden die Andeutungen, die mit dem Ort und der Zeit der Produktion betroffen sind, aus dem Medium abgeleitet. Im Rahmen der Ortspragmatik steht die Frage nach dem "Wo" im Vordergrund. Der Ort der Textproduktion und die Umgebung vom Sender und Textproduzent sind in Bezug auf die Ortspragmatik. Sprachliche und kulturell-politische Verhältnisse sind für die Ortspragmatik von Bedeutung, z.B. ein in einem Land mit Presse- oder Literaturzensur erscheinender Text muss "mit anderen Augen" gelesen werden als ein in einem Land ohne solche Beschränkungen erscheinender Text (Nord, 2009: 68). Die Zeitpragmatik spielt eine besondere Rolle zum Begreifen der Senderintention und auch bei der aktuellen Textsorten wie Pressemeldungen,-berichte, Wetterberichte usw. Denn der Übersetzer muss immer prüfen, ob die Informationen des Textes aktuell sind. Ausserdem betont Nord, dass die Zeitpragmatik sich also aus dem Erscheinungsdatum des Textes oder anderen Daten im Textumfeld ergeben kann und auch durch Recherchen über den Autor, sein übriges Werk und seine Intentionen bestimmt werden kann.

Wie oben angedeutet wurde, können Aufschlüsse mit Medium von der Orts- und Zeitpragmatik erlangt werden, weil das Zusammenspiel von diesen drei Pragmatiks eine erhebliche Rolle hat. Dieses Kinderbuch wurde erstmals im Jahr 1949 beim Europa Verlag in Zürich erschienen aber das im Rahmen dieser Arbeit analysierende Buch ist am Februar 2000 beim Deutscher Taschenbuch Verlag als 8. Auflage in München veröffentlicht. Der Europa Verlag wurde 1933 gegründet, er bezweckt ein Forum für verfolgte Autoren und verbotene Manuskripten zu bieten. ${ }^{6}$ Deutscher Tachenbuch Verlag ist auch 1960 als reiner Taschenbuchverlag für Zweitveröffentlichungen gegründet, aber heutzutage ist er als dtv Label bekannt und er veröffentlicht jährlich etwa 400 Titel, unter denen es internationale deutschsprachige Belletristik, Sachbücher, Kinder- und Jugendbücher usw. gibt.7 Ausserdem soll man die schritliche Angabe "In neuer Rechtschreibung" auf der 8. Auflage in Betracht ziehen, damit zeitgenösische Kinder durch neue Rechtschreibung dieses Buch erfassen können.

\section{Kommunikationsanlass und Textfunktion ${ }^{8}$}

Der Kommunikationsanlass eines Textes ist nich so leicht ausfindigzumachen. Durch den Sender, die Senterintention, die Empfänger und ihre Erwartungen kann man sich eine Idee von dem Kommunikationsanlass machen. Ausgehend von diesen Nords Auffasungen kann man auch keinen konkreten Anlass für diese Veröffentlichung ausmachen, aber weil dieses Kinderbuch im Jahr 1949 nach dem zweiten Weltkrieg von Kästner verfasst wurde, kann man sich daraus ergeben, dass zerstörende Ergebnisse des Krieges dargelegt werden. Und in diesem Buch wird bezweckt, ein gewaltfreies Leben und eine friedliche Zukunft durch die Tiereswelt zu bilden.

\footnotetext{
https://www.europa-verlag.com/ber uns.html, (abgerufen am 06. April 2021) https://www.dtv.de/verlag/ueber-uns-about-us/c-39, (abgerufen am 06.April 2021)

Textfunktion und Übersetzungstypen werden unter vorherigem Titel ausführlich erklärt, um die Bedeutung der Textfunktion im Rahmen dieser Arbeit zu bestimmen. Denn in dieser vorliegenden Arbeit ist es so wichtig, das Kinderbuch "die Konferenz der Tiere" im Hinblick auf die Übersetzungstypen nach Nord festzulegen, in welchen Übersetzungstypen bewertet werden soll.

Adres $\quad$ Address

RumeliDE Journal of Language and Literature Studies Osmanağa Mahallesi, Mürver Çiçeği Sokak, No:14/8 Osmanağa Mahallesi, Mürver Çiçeği Sokak, No:14/8 Kadıköy - İSTANBUL / TÜRKIYE 34714 Kadıköy - ISTANBUL / TURKEY 34714 e-posta: editor@rumelide.com e-mail: editor@rumelide.com tel: +90 505 7958124, +90 216773 o 616 phone: +90 505 7958124, +90 216773 o 616
} 
Nord betont, dass ein Autor meistens der Sender eines literarischen Textes ist, den Text selbst produziert und seine Intention nicht eine verbindliche Darstellung der Realität ist. Zusätzlich richtet sich der Text an einen bestimmten Adressaten und das Medium ist im Allgemeinen schriftlich. Nord macht die Übersetzungstypen als dokumantarische und instrumentalische Übersetzung klar, dass die wörtliche, philologische und exotisierende Übersetzung zum Typ der dokumentarischen Übersetzung und die funktionskonstante, funktionsvariierende und korrespondierende Übersetzung zum Typ der instrumentellen Übersetzung gehören. Und die Textfunktion muss aus der Konstellation der textexternen Faktoren erfasst werden, wenn es sich um keine Textortenbezeichnung handelt. Dieses fiktives Buch als dokumanterischer Übersetzungstyp ist ein literarischer Text, der die Kinder sowohl zur Unterhaltung als auch zum Nachdenken bringt.

\subsection{Textinterne faktoren des ausgangstextes}

\section{Textthematik}

Ausgehend von sechs Gründen stellt Nord die Wichtigkeit der Thematik für Translation fest.

1.Der este Grund ist die Textkombination, die aus der Hierarchie kompatibler Themen und Teilthemen, Ermittlung der unterchiedlichen Themen besteht.

2. Mögliche Präsuppositionen ergibt man sich aus der Thematik in einen bestimmten kulturellen Kontext.

3. Eingrenzung der Redegegenstände durch die Thematik wendet sich die Translatoren auf das Fachgebiet zu, um das notwendige Fachwissen zu haben und zu übersetzen.

4. Die Analyse der Thematik spielt eine besondere Rolle zur Feststellung der Vereinbarkeit der Ausgangstextbesonderheiten und des Übersetzungsauftrags.

5. Die Titelsfunktion dient zur thematischen Kennzeichnung des Textes.

6. Schliesslich gibt das Textthema bisweilen Hinweise auf textexterne Faktoren oder die textexternen Faktoren werden durch die Themabestimmung beweist (Nord, 2009: 94-95)

Unter den obengegebenen Informationen muss Textthematik in die Analyse miteinbezogen werden. Die Feststellung der Textthematik spielt eine erhebliche Rolle, um Aufschlüsse mit den anderen textinternen Faktoren zu erhalten. Der Titel des Buches "Die Konferenz der Tiere" bildet eine Auffasung über das Thema des Textes. Durch die Konferenz bezwecken alle Tiere auf der Erde Frieden zu schliessen und eine Welt ohne Krieg zu bilden, aber es geht nur um die Kinder, weil die Menschen durch die Kriege die Zukunft der Kinder zerstören. Neben diesem zentralen Thema handelt es sich um die Nebenthemen wie Freundschaft, Menschenverstand, Liebe, Kameradschaftsgeist usw.

\section{Textinhalt}

Zur Analyse des Textinhalts von syntaktischen oder semantischen komplexen Texten schlägt Nord vereinfachende Paraphrase als Verfahren vor, aber sie hebt hervor, dass der unparaphrasierte Ausgangstext immer als Grundlage für Übersetzung betrachtet werden muss. Kohäsionsmerkmale des Textes, Konnotation und Sekundärinhalt stehen im Vordergrund der inhaltlichen Textanalyse. Unter diesem Blickwinkel wird von den Inhalten des Kinderbuches kurzzusammenfassend erwähnt. Die Tiere sorgen sich um die Kinder, weil die Menschen immer wieder ergebnislosse Konferenze zum Weltfrieden organisieren. Eines Tages ist eine Idee dem Elefant, Oskar, eingefallen und er beginnt seinen kleinen Neffe- das Tapir Theodor- in Südamerika, das Känguru Gustav in Australien, den Eisbär Paul am Nordpol, die Eule Ulrich in Mitteleuropa, die Maus Max in Asien und schliesslich den

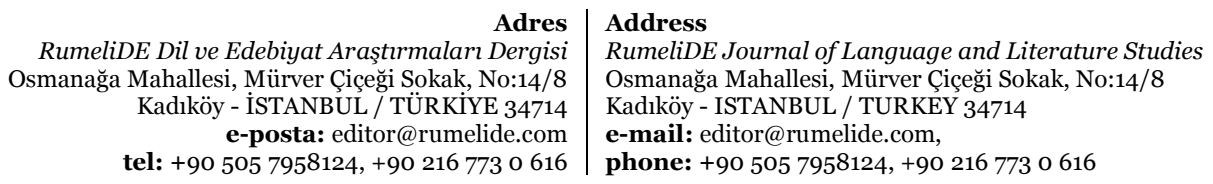


Text analysis for translation and translation problems in a children's book example:Erich Kästner's Animal Meeting / Ü. Albiz (pp. 685-707)

Stier Rheinhold in Nordamerika anzurufen. Er erklärt, was seine Idee ist und schlägt vor, eine erste und letzte Konferenz abzuhalten. Von nun an beginnt diese Nachricht unter den Tieren angekündigt zu werden: "Heute in vier Wochen Konferenz im Hochhaus der Tiere". Alle Tierorten auf der Welt wählen die Delegierten und sie bereiten sich sorgfältig für diese Konferenz vor. Am Tag der Konferenz stehen alle Delegierten und fünf Ehrengäste von Kindern in allen Farben zur Verfügung im Hochhaus der Tiere. Aber am gleichen Tag halten die Staatsmänner eine Konferenz in Kapstadt ab. Die Tiere zielen durch diese erste und letzte Konferenz auf einen Kompromiss unter den Menschen -Staatsmännernab, die trotz der vielen Konferenzen keinen Kompromiss über die Kriege haben. Die Tiere bringen präzis ihre Wille zum Ausdruck:

"Wir sind hier zusammengekommen, um den Kindern der Menschen zu helfen. Warum? Weil die Menschen selber diese ihre wichtigste Pflicht vernachlässigen! Wir verlangen einstimmig, dass es nie wieder Krieg, Not und Revolutionen geben darf! Sie müssen aufhören!” (Kästner,2000: 60).

Trotz ihrer Wille möchten sich die Staatspräsidenten nicht darüber einig sein, deshalb müssen die Tiere in der Lage sein, die Konferenz der Menschen -Staatspräsidenten- dreimal zu unterbrechen. Erstens liegen alle Akten der Konferenzteilnehmer, der Kommisionen, Referenten usw. dank der Mäuse und Ratten in Fetzen am Boden, weil die Tiere denken, dass die Kriege wegen dieser Akten stattfinden. Zweitens verschwinden alle Uniformen auf dem gesamten Planeten dank der Motten. Weil diese zweie Angriffe scheitern, müssen die Tiere einen anderen Weg finden. Als die Konferenz der Staatsmännern drittes Mal unterbrach, sind die Kinder der Menschen spurlos verschwunden, weil die Geduld der Tiere erschöpft ist.

"Nirgends hörte man ein Kinderlachen, nirgends ein Weinen. Die Eltern und Lehrer und alle Erwachsenen waren allein auf der Erde.” (Kästner, 2000: 79).

Der dritte Plan der Tiere nötigt die Menschen und Staatspräsidenten zur Einigung, weil alle Menschen- Mütter, Väter, Omas, Opas- traurig und auswegslos sind. Die Tiere siegen und ein Vertrag wird unterschrieben, damit die Staatspräsidenten versprechen, dass sie den Friede auf aller Welt bis zur Ewigkeit bewahren, alle Grenzen unter den Ländern abschaffen und ihre andere Wünsche erfüllen müssen.

\section{Präsuppositionen}

Weil es bei den Präsuppositionen per definitionem um das Nicht- Verbalisierte, Nicht-Gesagte geht, sind sie im Text nicht einfach zu ermitteln (Nord, 2009: 109). Bestimmend die Hintergrundinformation des Ausgangstextes können die Präsuppositionen abgeleitet werden. Unter diesem Titel entsteht der Begriff "Redundanz", unter dem Erläuterungen, Wiederholungen, Neuformulierungen, Zusammenfassungen versteht werden. Ausserdem sind die Präsuppositionen die Voraussetzungen, die Sprecher oder Autor in Bezug auf den Horizont ihrer Adressaten annehmen (Nord,2009: 108). Nord betrachtet, dass Sprecher oder Leser Präsuppositonen rekonstruierend verstehen können.

Unter den obengegebenen Informationen versucht man die Präsuppositionen dieses Werkes zu analysieren. Weil die Präsuppositionen ausgehend von der Biographie des Verfassers, den Textsorten, der Ideologie, der Religion und Kultur oder Politik eines Zeitabschnitts festgesetzt werden können, kann man sagen, dass Kästner, der Autor dieses Werkes, die Weltkriege erlebt hat und zerstörerische Ergebnisse der Weltkriege die Themen seiner Werke beeinflusst hatten. Er ist der bekannte Verfasser der Kinderliteratur. Um dieses Kinderbuch zu verstehen, bedürft man nicht ein umfangreiches

\footnotetext{
\begin{tabular}{r|l} 
Adres & Address \\
RumeliDE Dil ve Edebiyat Araşttrmaları Dergisi & RumeliDE Journal of Language and Literature Studies
\end{tabular} Osmanağa Mahallesi, Mürver Çiçeği Sokak, No:14/8 Osmanağa Mahallesi, Mürver Çiçeği Sokak, No:14/8 Kadıköy - İSTANBUL / TÜRKIYE 34714 Kadıköy - ISTANBUL / TURKEY 34714 e-posta: editor@rumelide.com e-mail: editor@rumelide.com tel: +90 505 7958124, +90 2167730616 phone: +90 505 7958124, +90 2167730616
} 
Hintergrundwissen. Falls Kinderleser über den Krieg und die Zerstörüng des Krieges im Bilde sind, können sie das Thema leicht begreifen. Wenn die Zeitepoche, in der das Werk verfasst wurde, besonders vor Augen gehalten wird, verstehen die Ausgangsempfänger oder -leser den Krieg und sein Thema mühelos, weil seine Ergebnisse noch auf der ganzen Welt behersschen. Auf diese Weise können Kinderleser erfassen, warum die Tiere sich um die Kinder sorgen und sie bewahren wollen.

\section{Aufbau und Gliederung des Textes}

Die Gliederung des Textes hinsichtlich der Makro- und Mikrogliederung ist wichtig für die übersetzungsrelevante Textanalyse. Denn die Feststellung der verschiedenen Teiltexten und der unterschiedlichen Funktionen ist von Bedeutung, um unterschiedliche Übersetzungsstrategien, bestimmte Gliederungskonventionen, Hinweise auf Textsorten und Textfunktion und die inhaltlichen Mikrostrukturen der komplexen und inkohärenten Texten zu bestimmen.

Das Werk "Die Konferenz der Tiere" hat keine verschiedene Teiltexte und kein Kapital. Der Ausgangstext besteht aus 100 Seiten, auf der Rückseite des Buches gibt es eine aus den 10 Zeilen bestehende Inhaltsangabe, auf der Vorderseite steht eine Illustration von der Konferenz der Tiere. Auf der ersten Seite dieses Ausgangstextes handelt man von der Biographie des Verfassers und des Illustrators, Walter Trier, wer Prager Zeichner und Kinderbuchillustrator ist. Unter dem Titel auf der zweiten Seite fällt der Ausdruck "nach eine Idee von Jella Lepman" ins Auge. Auf den letzten Seiten des Buches stehen Aufschlüsse und Bilder in Bezug auf die Werke von Erich Kästner.

\section{Nonverbale Textelemente}

Nonverbale Elemente sind sprachbegleitende oder -komplementäre Mittel und dienen zur Ergänzung, Verdeutlichung, Disambiguierung oder Intensivierung der Textaussage. Nonverbale Textelemente wie Layout, Gestik, Tabellen oder Bild im Comic kann man während der Ausgangstextanalyse bestimmen. Nord betont, dass in der mündlichen realisierten Kommunikation gestische, mimische Signale und Körperbewegungen unter diesem Begriff bezeichnet werden können. Die einigen Faktoren -besonders Senderintention und Textfunktion- von den textexternen Faktoren werden durch nonverbale Textelemente gekennzeichnet.

Weil dieses Werk als Textsort ein Kinderbuch ist, werden Illısturationen fast auf jeder Seite beobachtet, die spezifisch nach dem Inhalt jeder Seite gezeichnet werden. Durch diese Bilder können die Kinder die Handlungen, die Charaktere und ihre Welt begreifen.

\section{Lexik und Syntax}

Die in einem Text sowohl von textexternen als auch von textinternen Faktoren bestimmte Wortwahl deutet auf die semantische und stilistische Merkmale der Lexik wie Konnotatiton, Wortfeldzugehörigkeit hin und gibt Afschlüsse über Inhalt, Thematik und grammatisch-formale Charakteristika. Im Hinblick auf diese Meinungen von Nord wird den Faktor "Lexik" im Ausgangstext untersucht.

Falls die Kinder als die Zielgruppe oder Adressaten dieses Werkes angenommen werden, ist es möglich zu behaupten, dass sein Verfasser, seine Wortwahl, seine Schreibart oder seinen Stil im Rahmen von dem Relativitätsprinzip bestimmt werden. Weil die Charaktere des Textes aus den Tieren bestehen, kann vorausgesetz werden, dass die Kinder die Namen der Charaktere kennen. Ausserdem hat jedes

\footnotetext{
Adres $\mid$ Address

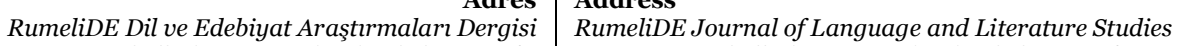
Osmanağa Mahallesi, Mürver Çiçeği Sokak, No:14/8 Osmanağa Mahallesi, Mürver Çiçeği Sokak, No:14/8 Kadıköy - İSTANBUL / TÜRKIYE 34714 Kadıköy - ISTANBUL / TURKEY 34714 e-posta: editor@rumelide.com e-mail: editor@rumelide.com, tel: +90 505 7958124, +90 2167730616 phone: +90 505 7958124, +90 2167730616
} 
Text analysis for translation and translation problems in a children's book example:Erich Kästner's Animal Meeting / Ü. Albiz (pp. 685-707)

Tier ein konkretes Personlichkeitsmerkmal, weil der Verfasser die Tiere mit einem Eigennamen benennt.

Nord (2009: 132) erklärt, dass die Syntax Aufschluss über Inhalt, Thematik und Aufbau des Textes, suprasegmentale Merkmale gibt und die durch die Syntax gekennzeichnenden textexternen Faktoren besonders Senderintention, Medium und Textfunktion sind. Als Kennzeichnungen der Syntax in einem Text sind Satzlänge, Satzformen wie Aussagesätze, Fragesätze, Ausrufe und satzwertigen Strukturen wie Infinitiv- Parttizipalkonstruktionen und Haupt-und Nebensätze zu analysieren. In dem Ausgangstext geht es um kurze, einfache Sätze und manchmal auch um die Redewendungen. Dadurch kann den Rückschluss auf die Senderintention- den Verfasserintention- gezogen werden, dass der Verfasser bewusst seine Zielempfänger orientiert und verständlich sein möchte.

\section{Suprasegmentale Merkmale}

Nord bestimmt, dass man als suprasegmentale Merkmale eines Textes die Merkmale seiner Gestaltung betrachtet, die über die segmentalen Einheiten der Lexik und Syntax hinaus gehen und die Analyse der suprasegmentalen Merkmale des Textes Aufschlüsse auf den Inhalt (z.B. ironische Betonung) und die Thematik (z.B. feierlicher Tonfall bei einer Rede), auf die Präsuppositionen (z.B. Abbruch des Intonationsbogens bei einer Anspielung) und den Aufbau (z.B. Pausen, Betonung der rhematischen Satzteile) zulässt (Nord, 2009: 139). Sender, Senderintention, Ort, Anlass und Textfunktion als textexterne Faktoren spielen eine grosse Rolle zum Bestimmen der suprasegmentalen Merkmale.

Der Ausgangstext als suprasegmentale Merkmale beinhaltet manchmal Ausrufe- und Anführungszeichen und einige Telegrafnachrichten, die kurze und lange Striche und Besonderheiten einer Telegrafnachricht enthalten.

telegram an alle welt:-..-konferenz in london beendet-..-verhandlungen ergebnislos-..- (Kästner, 2000: 5).

Während die Gefühle wie Aufregung, Wut durch die Ausrufezeichen wiederspiegelt werden, werden Anführungszeichen für bilaterale Dialoge verwendet.

"O diese Menschen! Wenn ich nicht so blond wäre, könnte ich mich auf der Stelle schwarz ärgern!" (Kästner, 2000: 5).

"Schon gut”, unterbrach ihn die Giraffe. “Aber Schimpfen hilft nichts. Es müsste etwas geschehen”

"Jawohl!", trompetete Oskar, der Elefant. "Vor allem wegen der Kinder, die sie haben- aber was?" (Kästner, 2000: 7).

Im ganzen Buch handelt es sich nicht um einen Fettdruck sondern um drei Kursivdrücke. In diesem Text werden die Kursivdrücke zur Hervorhebung verwendet.

"Wir verlangen einstimmig, dass es nie wieder Krieg, Not, Revolution geben darf! Sie müssen aufhören! Denn sie können aufhören! Und deshalb sollen sie aufhören!” (Kästner, 2000: 60).

\subsection{Wirkung}

Nord betont, dass den Wirkungsbegriff hinsichtlich des Empfängers beobachtet werden soll, weil der Empfänger den Text vor dem Hintergrund seiner Erwartungen rezipiert und sein Hintergrundwissen im Vordergrund steht. Aber Nord erregt Aufmerksamkeit über die historische Wirkung, die bei der Analyse einer älteren Textübersetzung wie Bibel, Odyssee unter der Zeitpragmatik zugerechnet werden

\footnotetext{
\begin{tabular}{r|l} 
Adres & Address \\
RumeliDE Dil ve Edebiyat Araşttrmaları Dergisi & RumeliDE Journal of Language and Literature Studies
\end{tabular} Osmanağa Mahallesi, Mürver Çiçeği Sokak, No:14/8 Osmanağa Mahallesi, Mürver Çiçeği Sokak, No:14/8 Kadıköy - ISTANBUL / TÜRKIYE 34714 Kadıköy - ISTANBUL / TURKEY 34714 e-posta: editor@rumelide.com e-mail: editor@rumelide.com, tel: +90 505 7958124, +90 2167730616 phone: +90 505 7958124, +90 2167730616
} 
muss. Im Rahmen der zentralen Faktorenrelationen hebt Nord hervor, dass die Senderintention ein wichtiges Gewicht hat, um die Wirkung zu steuern. Unter dem Titel "Relation II: Empfänger-Textwelt" bestimmt sie, dass für die Empfänger die Darstellung der Textwelt auf eine durch Vorwissen, Horizont und Gestimmtheit bedingte Erwartung trifft.

Wie oben angedeutet wurde, wird der Begriff "Wirkung" in diesem folgenden Abschnitt hinsichtlich des Ausgangstextes analysiert. Wenn ein Empfänger dieses Buch von Kästner festhält, prägen sich seine Erwartungen gemäss einem Kinderbuch aus. Wenn dieses Buch hinscihtlich der Zeitpragmatik bewertet wird, behauptet man, dass es noch ein aktuelles Buch wegen seines Themas ist, obwohl das Buch im Jahr 1949 veröffentlicht wurde. Weil die Themen wie Kinder, Liebe, Frieden, Bewahrung im Vordergrund stehen, haben diese Begriffe auf die Kinder einen wichtigen Einfluss noch in unserer Zeit. Konventions- oder Kulturbezogenene Wirkung hat keine Abweichung, weil sein Thema nicht von deutscher Kultur oder deutschen Konventionen abhängt.

\subsection{Zieltextanalyse}

Die gleichen Faktoren wie in der Ausgangstextanalyse werden in Bezug auf türkische Übersetzung analysiert. Um textexterne und textinterne Faktoren zu bestimmen, ist die Zielsituation von Bedeutung, weil das Ziel sich in einer anderen Situation verändert. Der Translationsvorgang wird startet, wenn sich ein Initiator an einen Übersetzer wendet. Denn ein bestimmter Zieltext wird für einen bestimmten Rezipienten benötigt oder der Initiator / Auftraggeber will einen Text, der in einer Ausgangssprache von einem Textproduzenten verfasst oder von einem Textsender gesendet wird, in der Zielsprache lesen (Nord, 2009: 5)

Nord erklärt ausführlich, wie der Translationsvorgang anfängt und welche Faktoren während dieses Vorgangs Rolle spielen.

\subsubsection{Textexterne faktoren}

\section{Senderpragmatik und Senderintention}

Der türkische Sender des Werkes von Kästner ist der Verlag Can Çocuk 9 und der hat der Übersetzerin Süheyla Kaya Übersetzungsaufgabe gestellt, damit sie das erwähnte Kinderbuch ins Türkische übersetzen könnte. Der Verlag Can Çocuk wurde 1981 von Erdal Öz gegründet und veröffentlicht seither nationale und internationale Kinderbücher. Auf der Webseite des nur kinderorientierte Bücher veröffentlichenden Verlags wird zum Ausdruk gebracht, dass der Verlag bezweckt, den Kindern mit verschiedenen Tätigkeiten Spass zu machen und sie mit den Autoren zusammenkommenzulassen. Aus diesen Ausdrücken kann die Senderintention abgeleitet werden. Um den Übersetzungsprozess klarzumachen, Kenntnisse über den Übersetzer/ der Übersetzerin zu haben, ist erforderlich. Auf dieser türkischen Fassung steht nur der Name der Übersetzerin, aber es gibt noch keine weitere Information. Süheyla Kaya übersetzte viele Werke von bekannten Autoren wie Peter Handke, Elfriede Jelinek, Renus Berbig, Judit Kuckart, Reiner Maria Rilke usw. 


\section{Empfängerpragmatik}

Zielempfängerkreis wie in dem Ausgangsempfängerkreis zielt auf die 8-10 jährigen Kinder, dass diese Information mit Empfänger auf der Rückseite des Romanes angedeutet wird.

\section{Medium/Kanal, Orts- und Zeitpragmatik}

Die erste türkische Ausgabe vom Buch "die Konferenz der Tiere" erschien 1981 beim Can Çocuk Verlag und siebzehnte Ausgabe, die im Rahmen dieser Arbeit analysiert wird, wurde am April 2018 beim Can Çocuk Verlag in İstanbul veröffentlicht. Heutzutage erreicht dieses Werk noch hohe Verkaufzahlen und türkischer Leserkreis fordert es gern.

\section{Kommunikationsanlass und Textfunktion}

Einen konkreten Kommunikations- oder Publikationsanlass zu bestimmen, ist natürlich unmöglich. Aber weil es sowohl als ein fiktives Werk in dem literarischen Bereich als auch nach dem zweiten Weltkrieg verfasst wurde, kann man darauf Rückschlüsse ziehen. Im Grunde genommen bezweckt dieser Roman sowie jede Literarische Werke die Leserschaft zu vergnügen, nachdenken zu bringen usw. Aber der Can Çocuk Verlag legt die Themen wie Vorstellungskraft, Liebe, gesellschaftliches Leben und Unterthemen wie Frieden, Kampf, Krieg und seine Zerstörüngen usw. fest, deshalb wird daraus abgeleitet, wie der Text eine Funktion übernimmt und tiefe literarische Bedeutung unter den Wörtern steht.

\subsubsection{Textinterne faktoren}

Textthematik und Textinhalt des Zieltextes sind identisch wie im Ausgangstext. Deshalb werden von diesen Faktoren unter diesem Titel wieder nicht gehandelt.

\section{Präsuppositionen}

Die für die Ausgangstextanalyse gegebebenen Aufschlüsse sind gültig auch für die Präsuppositionen der Zieltextanalyse. Der Text kann von den Zieltextlesern leicht begriffen werden, weil der Text ein globales Thema behandelt und kein kulturelles Hintergrundwissen braucht. Möglicherweise hat Zieltextempfänger Vorwissen über den Krieg durch die andere Kinderbücher oder Cartoonfilme usw.

\section{Aufbau und Gliederung des Textes}

Der Zieltext hat 84 Seiten. Obwohl Zieltexts Seitenzahl im Vergleich zu der Seitenzahl des Ausgangstextes geringfügig ist, benutzt die Übersetzerin keine umfangreichen Reduktionsstrategie oder Verkürzungsstrategie ausser zwei oder drei Sätzen. Auf der ersten Seite des Zieltextes wird die Reise der Tiere für die Konferenz gezeichnet, auf der Rückseite wird nicht eine Inhaltsangabe wie im Ausgangstext sondern den Hauptgedanke mit drei oder vier Sätzen abgefasst und eine ganz wichtige Frage wird gestellt: "Wer liebt die Kinder am meisten?" Und auf der ersten Innenseite gibt es nur die Biographie des Verfassers und keinen Bescheid über die Übersetzerin. Der Name der Übersetzerin steht nur auf der Vorderseite mit kleinem Druck.

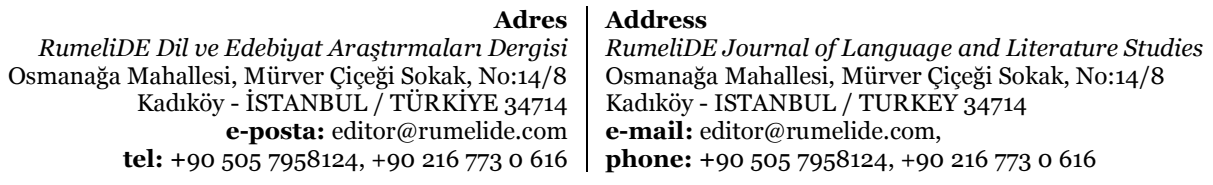

RumeliDE Dil ve Edebiyat Araşttrmaları Dergisi i, Mürver Çiçeği Sokak, No:14/8 e-posta: editor@rumelide.com tel: +90 505 7958124, +90 2167730616
Address

Osmant Journal of Language and Literature Studies

(

phone: +90 505 7958124, +90 2167730616 


\section{Nonverbale Textelemente}

Der Zieltext hat gleiche Bilder oder Illustruationen von Walter Trier und diese sind auf jeder Seite. Dank dieser Bilder können sich Zieltextleser oder Kinderleser die Handlungen, Tiere und ihr Aussehen und schliesslich alles vorstellen, was in dem Text passiert ist. Durch diese Bilder können Zieltextleser das Geheimnis des Textes dekodieren und durch sie wird der Text klar gemacht.

\section{Lexik und Syntax}

Während der Autor als Sender in nicht-fiktionalen Texten meistens selbst entsteht, zeigt sich ein implizierter Erzähler. Deshalb kann man sagen, dass die Wortwahl die Senderintention wiederspiegelt. Diese Äusserung von Nord deutet auch auf die Intention der Übersetzerin in diesem Zieltext, weil sie versucht, die Schreibweise und den Stil des Verfassers zu bewahren. Die Übersetzerin hat auch einfach verständliche Wörter gewählt und Eigennamen der Tiere wie Alois, Oskar, Leopold usw. ins Türkische gleich übertragen. Verwendend fremdartige Elemente in dem Zieltext schafft sie, dass der Zieltextleser eine Image hat, ob er einen fremden Text oder ein übersetztes Werk liest. Satzstrukturen sind kurz, einfach und verständlich.

\section{Suprasegmentale Merkmale}

Um die suprasegmantelen Merkmale in dem Zieltext zu bestimmen, müssen suprasegmentale Merkmale des Ausgangstextes beachtet werden. Dadurch kann man feststellen, ob die Übersetzerin oder der Übersetzer Achtung auf die suprasegmantelen Besonderheiten des Ausgangstextes hat. In diesem genannten Zieltext wird festgestellt, dass die Übersetzerin den Ausgangstext als Grundlage nimmt und gleiche suprasegmentale Merkmale durch die Übersetzung in die türkische Version überträgt. Die Besonderheiten der Telegrafnachrichten werden behalten und Ausrufe- oder Anführungszeichen wie im Ausgangstext werden verwendet.

bütün dünyaya telgraf: -.- londra konferansı sona erdi -..- (Kästner, 2018:8).

Ausrufe- und Anführungszeichen bedienen für gleiche Ziele sowie im Ausgangstext.

“Ah şu insanlar! Ĕger bu kadar sarışın olmasaydım, şu an öfkeden mosmor kesilebilirdim!” (Kästner, 2000:8).

Ausserdem verwendet die Übersetzerin auch drei Kursivausdrücke für gleichebedeutende Verben.

"Biz savaşların, açlıkların ve devrimlerin, bir daha asla yaşanmamasını oy birliğiyle talep ediyoruz! İnsanlar buna bir son vermek zorundalar. Son verebilirler! Son vermeliler!” (Kästner, 2018:52).

\subsection{Wirkung}

Nord bestimmt, dass die Auswahl der Inhalte bei fiktionalen Texten besondere Wirkung hat. Bei diesen fiktionalen Texten hat der Sender aus einer endlosen Vielfältigkeit möglicher Informationen zu einer Person oder einem Ereignis ausgewählt. Dadurch erzielt er, die von ihm begehrte Wirkung zu machen. Die Auswahl des Themas oder der Inhalte muss von der Übersetzerin oder dem Übersetzer in dem Zieltext oder in dem übersetzten Werk bewahrt werden, um die von dem Sender oder Verfasser intendierten Einfluss auch auf Zielempfänger oder Zieladressaten haben zu können. Für die Translation von Texten ist diese Relation zwischen dargestellter Textwelt und Empfängererwartung von besonderer Bedeutung, da in jedem Fall eine kulturelle Distanz, in den allermeisten Fällen eine 
Text analysis for translation and translation problems in a children's book example:Erich Kästner's Animal Meeting / Ü. Albiz (pp. 685-707)

räumliche und in sehr vielen Fällen auch eine gewisse zeitliche Distanz zu überwinden ist (Nord, 2009: 150). In diesem türkischen Zieltext kann man behaupten, dass die Übersetzerin die genannte Relation zwischen dargestellter Textwelt und Empfängererwartung wiederspiegeln kann. Denn wenn man angenommen wird, dass die Zielempfänger oder Zielleser die türkischen oder türkissprachigen Kinder sind, kann man die Ansicht vorbringen, dass der Zieltext hinsichtlich des Themas, der Lexik oder Syntax die beabsichtigten Ziele erreicht. Und weil der Ausgangstext keine grossen temporalen oder konventionellen Hindernisse im Wesentlichen habt, muss die Übersetzerin diese Hindernisse nicht überwinden. Natürlich gibt es sprachenpaarbezogenene Unterschiede wie Konnotationen, Redewendungen, Alliterationen, die Übersetzerin Süheyla Kaya erfolgreich überwunden konnte.

\section{6. Übersetzungsprobleme des zieltextes am beispiel des werkes "die tiere der konferenz"}

\section{Pragmatische Übersetzungsprobleme (PÜP)}

Nord macht deutlich, dass die pragmatischen Übersetzungsprobleme aus den pragmatischen Faktoren besonders textexternen Faktoren ergeben. Die Titel oder Überschrifte zu übersetzen ist ein pragmatisches Problem hinsichtlich der Textfunktion und nach Nord entstehen pragmatische Übersetzungsprobleme in jeder Translationsaufgabe. Wenn unter diesem Blickwinkel die türkische Übersetzung im Hinblick auf die pragmatischen Übersetzungsprobleme untersucht wird, kann zuerst der Titel analysiert werden. Während der Titel des Ausgangstextes "Die Konferenz der Tiere" ist, ist der Titel der türkischen Version "Hayvanlar Toplantısı." Aber in der türkischen Version ist es auch möglich den Titel als "Hayvanlar Konferansı" zu übersetzen. In der Analyse dieses Zieltextes können keine erheblichen pragmatischen Übersetzungsprobleme wegen der textexternen Faktoren bestimmt werden, da Sender-, Empfänger-, Orts-, Zeitpragmatik sowohl in dem Ausgangstext als auch Zieltext bekannt sind. Natürlich geht es um spezifischen Kontrast vom Ausgangsempfänger und Zielempfänger, aber weil Textthematik und Texinhalt noch aktuell und gemäss den Kindern sind, können sie diesen Kontrast beseitigen.

\section{Konventionsbezogene Übersetzungsprobleme (KÜP)}

Wenn man unterschiedliche Verhaltensweisen in der Ausgangs- und Zielkultur erwartet, enstehen aus diesem Grund Übersetzungsprobleme. Textsorten, Mass-, Stil- und Übersetzungskonventionen gehören zu den konventionsbezogenen Übersetzungsproblemen (Nord, 2009: 178). Ausgehend von konventionsspezifischen Problemen von Nord behauptet man, dass der Ausgangstext wenige kulturspezifische Besonderheiten trägt. Deshalb enstehen keine wichtige Probleme. Aber z.B. Eigennamen der Tiere oder Orten werden in dem Zieltext wie im Ausgangstext zum Ausdruck gebracht. In dem Ausgangstext sind die Eigennamen der Tiere, der Orten oder die Gattungsnamen sowie der Elefant Oskar, der Löwe Alois, die Giraffe Leopold, der Löwe Hasdrubal; Vietnam; SaharaAbendblatt usw. In dem Zieltext werden die Eigennamen der Tiere, der Orten oder die Gattungsnamen gleich übertragen: Fil Oskar, Aslan Alois, Zürafa Leopold, Aslan Hasdrubal; Vietnam; Sahara-Akşam Gazetesi. Die Stadt Kapstadt von Südafrika wird ins Türkisch als Cape Town übersetzt, und ein Wort "Toupet" io in dem Ausgangstext wird von Süheyla Kaya als Toupet übertragen. Dieses Wort bildet ein Problem in der Verstehensphase des Zieltextlesers. Denn der Zielleser muss es recherchieren oder nachfragen, aber der Zielempfängerkreis besteht besonders aus den Kindern, deshalb die Kinder können nicht diese Verstehungs- oder Übersetzungsprobleme überwinden. Weil die Übersetzerin für

$10 \quad$ In dem Ausgangstext steht das Wort auf der Seite 91; in dem Zieltext steht es auf der Seite79.

Adres $\mid$ Address

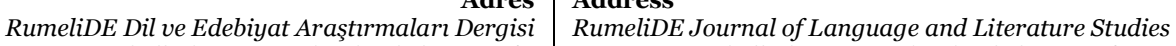

Osmanağa Mahallesi, Mürver Çiçeği Sokak, No:14/8 Osmanağa Mahallesi, Mürver Çiçeği Sokak, No:14/8

Kadıköy - İSTANBUL / TÜRKIYE 34714 Kadıköy - ISTANBUL / TURKEY 34714

e-posta: editor@rumelide.com e-mail: editor@rumelide.com,

tel: +90 505 7958124, +90 2167730616 phone: +90 505 7958124, +902167730616 
einen spezifischen Empfängerkreis übersetzt, muss sie diese Übersetzungsprobleme entfernen. Die Übersetzerin kann "peruk" oder "takma saç" für türkische Version statt dieses Wortes "Toupet" verwenden.

"Steward, bitte einen Grog von Rum!" (Kästner,2000: 51).

"Bana bir sulu rom lütfen!” (Kästner, 2018: 51).

Ausgehend von diesen Ausdrücken wird bestimmt, dass das Wort "rom" in der Übersetzung in der Verstehensphase ein Problem bildet. Der 8-10 jährige Empfängerkreis kann nicht die Bedeutung des Wortes fassen. Obwohl dieses Wort in der türkischen Sprache immer wieder verwendet wird, kann dieses Wort einfach als "içki” ins Türkisch wegen der Zieladressaten übersetzt werden.

"O diese Menschen! Sie können nur zerstören! Sooft sie aufbauen wollen, wird’s ein Turm zu Babel!” (Kästner,2000: 11).

"Ah şu insanlar! Sadece yıkmayı biliyorlar. Ne zaman bir şeyler inşa etmek isteseler ortaya çıkan şey, insan gururunun utanç kaynağı bir Babil Kulesi oluyor!” (Kästner,2018: 13)

In diesem Abschnitt geht es auch um kulturelles Wissen, um zu verstehen, was Babel Turm ist. Aber dieses kulturelle Wissen gehört nicht zu einer Konvention oder Tradition, sondern zum Allgemeinwissen.

\section{Sprachenpaarbezogene Übersetzungsprobleme (SÜP)}

Sprachenpaarbezogene Übersetzungsprobleme treten immer wieder unter Sprachen auf, falls es um die Übersetzung geht. Die Verwendung von Indikativ oder Konjunktiv oder Hilfsverben usw. entsteht bei der Übertragung als Übersetzungsprobleme. Für solche Probleme gibt es keine bestimmten Lösungen (Nord, 2009: 178). Die Übersetzungskompetenz besonders Sprachkompetenz des Übersetzers oder der Übersetzerin spielen eine erhebliche Rolle, um diese Übersetzungsprobleme zwischen zwei Sprachen zu reduzieren oder zu überwinden. In diesem analysierten Zieltext und Ausgangstext entstehen die sprachenpaarbezogenen Übersetzungsproblemen im Vergleich zu den anderen Übersetzungsproblemen am häufigsten. Aber die Übersetzerin, die befriedigende Sprachkompetenz hat und die Nuance und Einzelheiten zweier Sprachen beherrscht, bietet ihren Ziellesern ein verständliches und lesbares Übersetzungsbuch an.

“Kriege!” knurrte der Löwe Alois. "Kriege bringen sie zustande. Und Revolutionen. Und Streiks. Und Hungersnöte. Und neue Krankheiten. Wenn ich nicht so blönd wäre, könnte ich mich auf der Stelle...” (Kästner, 2000: 6).

"Savaşlar! diye homurdandı Aslan Alois. "Sürekli savaşıyorlar. Devrimler ve grevler yapıyorlar. Her yerde açlık kol geziyor. Yeni hastalıklar da cabası. Ĕger bu kadar sarışın olmasaydım şu an..." (Kästner, 2018)

In dem vorgenannten Zitat bildet die Übersetzerin die Ausdrücke mit der Konjunktion "und" einen genauen Satz und sie bevorzugt eine Redewendung für das Wort "Hungersnote". Die Übersetzerin verwendet ihre Muttersprache effektiv. In ihrem Übersetzungsbuch verwendet sie viele Redewendungen. Auf diese Weise schafft sie auf den Lesern Einfluss zu haben. Manche Beispiele dafür lautet so:

"Es ist zum Ausderhautfahren" brummte er (Kästner, 2000: 10)."

"Zıvanadan çıkmak işten değil” diye homurdandı (Kästner, 2018:12).”

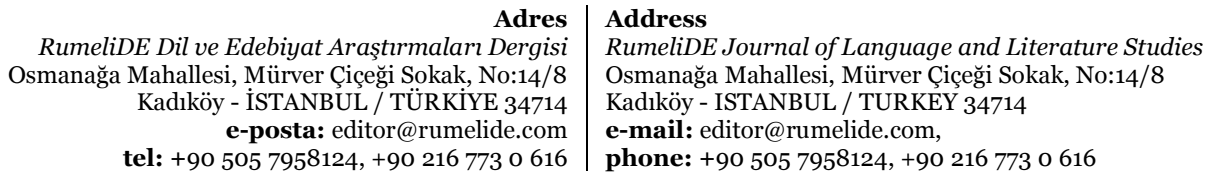


Text analysis for translation and translation problems in a children's book example:Erich Kästner's Animal Meeting / Ü. Albiz (pp. 685-707)

"Das ist eine Erdhälfte" sagte der Elefant zu seiner Frau. "Und überall herrschen unter den Menschen Not und Unvernunft" (Kästner, 2000: 12)

"Bu yarım kürelerden biri," dedi, "her yerde sefalet diz boyu, kimse sağduyulu davranmiyor (Kästner,2018: 13).”

"Da hatten die Störche und Flamingos, die im ägyptischen Hauptpostamt als Telefonfräuleins angestellt waren, mächtig zu tun (Kästner, 2000: 14)."

"Mısır Merkez Postanesi’nde çalışan leylek ve flamingoların işleri başlarından aşkındı (Kästner, 2018: 16)."

“Ja, Oskar!" antworteten die sechs, so laut sie konnten (Kästner, 2000: 15).”

"Evet, Oskar!” yanıtını verdi karşı taraftaki altı kişi; avazları çıktı̆̆ı kadar bağırmışlardı (Kästner, 2018: 17).”

"Das Schneehuhn wurde noch blasser, als es schon war (Kästner, 2000: 42)."

"Kar tavuğunun beti benzi iyice atmıştı (Kästner, 2018: 37)."

In dem Buch von Kaya gibt es noch viele Beispielsätze wie obengegebenen Sätze. Sie nutzt Verdoppelungen in der türkischen Version aus.

“....obwohl sie flach über den Wellen dahinflogen wie über den grünwogenden Wipfeln eines unendlichen Waldes (Kästner, 2000:49).”

"Dalgalar, uçsuz bucaksız bir ormanda, rüzgârda dalgalanan yeşil ağaç tepelerini andırıyordu (Kästner, 2018: 43).”

"Die Frösche hockten aufgeplustert in den Sümpfen und Teichen und quakten die Nachricht unermüdlich in die Lüfte (Kästner, 2000: 20).”

"Küçük göl ve bataklıklarda kabararak oturan kurbağalar da bu haberi bıkmadan usanmadan havaya virakladılar (Kästner, 2018: 21).”

Die Übersetzerin verwendet bisweilen Hinzufügen- und Auslassungsstrategie, deshalb kann man manche Abweichungen aufkommen.

"Ja, sogar die Schnecke Minna kroch aufgeregt aus ihrem Einfamilienhaus heraus und schleptte sich, das Haus auf dem Rücken, vor laute Atembeschwerden prustend und schnaufend, durch die Weinberge...( Kästner, 2000: 22)”

“Wir sind doch zwei Vögel!”, piepsten das Schneehuhn und der Pinguin (Kästner, 2000: 50).

Diese Sätze werden nicht von der Übersetzerin ins Türkische übersetzt. Den zugrunde liegenden Anlass zu bestimmen, ist nicht möglich. Aber weil es keinen konkreten Grund wie İdeologie, Politik, Religion in diesen Sätzen gibt, kann man vermuten, dass die Übersetzerin sie absichtsloss vergessen hat.

"Das ist die eine Erdhälfte" sagte der Elefant zu seiner Frau. "Und überall herrschen unter den Menschen Not und Vernunft (Kästner, 2000: 12)"

"Bu yarım kürelerden biri” dedi, "her yerde sefalet diz boyu, kimse sağduyulu davranmıyor. (Kästner, 2018: 14).

"Der Zahnarzt war ein Neger, schwarz wie Ebenholz und hatte einen kleinen Sohn mit grossem, runden Augen (Kästner, 2000: 27).”

“Abanoz gibi kara derili olan bu adamın, kocaman, yucarlak gözlü küçük bir de oğlu vardı (Kästner, 20018: 27).”

Die fettgedruckte Schreibweise in dem Zieltext werden von der Übersetzerin hinzugefügt, obwohl diese Sätze oder Wörter in dem Ausgangstext nicht vorhanden sind.

\begin{tabular}{|c|c|}
\hline es & \\
\hline RumeliDE D & dLiterature Studies \\
\hline a Mah & Mahallesi, Mürver Çiçeği Sokak, No:14/8 \\
\hline Kadıköy - İSTANBUL / & ISTANBUL / TURKEY 34714 \\
\hline $\begin{array}{l}\text { e-posta: editor@rumelide. } \\
\text { tel: }+905057958124,+902167730\end{array}$ & $\begin{array}{l}\text { editor@rumelide.com, } \\
\text { +90 505 7958124, +90 } 216773 \text { o } 616\end{array}$ \\
\hline
\end{tabular}


Durch die untengegebenen Beispiele wird bezweckt, sinngemässe Übersetzungen, Übertsetzungsfehler und Übersetzungvorschlagen anzubieten.

"Das ist die andere Erdhälfte" sagte der Elefant zu seiner Frau. Und überall herrschen seit Jahrhunderten Krieg, Not und Unvernunft. Das sieht jeder Mensch...(Kästner, 2000: 13)”

“ Bu da diğer yarım küre” diye devam etti. Yüzlerce yıldan bu yana insanlar sefalet içinde yaşıyor, sağduyu adeta unutulmuş. Bütün hayvanlar bunun farkında... (Kästner, 2018: 15).”

“Fil, bu da diğer yarım küre, dedi karısına. Buranın her yerinde, yüzyıllardan beri savaş, açlık ve sefalet var. Herkes bunun farkında...” (Ümmügülsüm Albiz, Übersetzungsvorschlag)

Wenn der Ausgangs- und Zieltext verglichen werden, wird festgelegt, dass es um einen Übersetzungsfehler geht. Der Ausdruck "Das sieht jeder Mensch" kann man ins Türkisch als "Herkes, bunun farkında" "Herkes, bunu biliyor" übersetzen.

Manche Beispiele durch die Übersetzungsvorschlagen zu geben, ist möglich, um sinngemässe und verständliche Übersetzungen zu erzielen. Die Übersetzung in dem ersten Beispiel kann nicht Gefühl der Wut genauso in dem Zieltext wiederspiegeln. Natürlich soll man annehmen, dass alle folgenden Übersetzungen richtig sind aber manche Lücken haben. Alternative Übersetzungen sind nur Vorschläge für bedeutungsvollere Übersetzungen.

“Dumme Gänse” brummte Oskar verärgert. “Gänse?” riefen die Telefonfräuleins empört. "Erlauben Sie mal! Hier werden nur Störche und Flamingos beschäftigt! (Kästner, 2000: 17).”

"Kaz Kafalılar" diye homurdandı Oskar öfkeli. "Kaz mı dediniz," diye bağrıştı santral memurları; kızmışlardı. "Şey özür dileriz ama burada sadece leylekler ve filamingolar çalışıyor! (Kästner, 2018: 19)”

Oskar, öfke ile "Kaz Kafalılar" diye homurdandı. "Kaz mı dediniz" diye sinirli bir şekilde bağırdı santral memurları. "Kusura bakmayın ama burada yalnızca leylekler ve filamingolar çalışıyor! (Ü.A., Übersetzungsvorschlag)"

"Sie hatten, als wären sie Briefträger, die wichtigste Post in ihren Beuteln. Und die Post lautete: "Heute in vier Wochen Konferenz im Hochhaus der Tiere! (Kästner, 2000: 21)"

"Postacılar gibi keselerinde taşıdıkları mesaj aynıydı: "Dört hafta sonra bugün Hayvanlar Gökdeleni'nde konferans var! (Kästner, 2018: 22).

"Onlar, çantalarında önemli haber taşıyan postacılar gibiydiler. Haber ise aynıydı: "Dört hafta sonra bugün, Hayvanlar Gökdeleni’nde konferans var (Übersetzungsvorschlag)”

\section{Text(exemplar)spezifische Übersetzungsprobleme (TüP)}

Das Werk Die Konferenz der Tiere ist ein literarischer Text, dessen Texttyp nach Reiss der senderorientierte expressive Text ist. Das Kennzeichen des expressiven Texttyps ist seine Senderorientiertheit, d.h. die individuelle künstlerische Prägung durch einen Autor. Wenn ein solcher Text übersetzt wird, so geht es insbesondere um die Vermittlung eines Sprach- oder Dichtkunstwerkes an eine zielsprache Gemeinschaft (Reiss, 1993: 21). Dieses Werk überträgt auch dieses Kennzeichen. Und es ist auch ein Kinderbuch, das besonders auf die Kinder gezielt ist. Die Kinderliteratur ist ein Sonderfall, der für die Kinderwelt, ihre Psychologie usw. geeignet sein muss. Unter Kinderliteratur kann man entweder Literatur verstehen, die für die Kinder geschrieben und intendiert ist, oder Literatur, die von Kindern gelesen wird, ganz gleich ob sie nun für Kinder gedacht war oder nicht (Oittinen, 1999: 251). Unter diesen Kenntnissen kann man behaupten, dass die Übersetzerin den Zieltext in Anbetracht der Kinder in dem Zieladressatenkreis übersetzt. Die Einstellung des Sendersdes Verfassers- des Ausgangstextes deckt sich mit der Einstellung des Senders- des Verlags oder der Übersetzerin-. Deshalb tretten nicht besondere textspezifische Übersetzungsprobleme auf.

\begin{tabular}{|c|c|}
\hline es & \\
\hline RumeliDE D & dLiterature Studies \\
\hline a Mah & Mahallesi, Mürver Çiçeği Sokak, No:14/8 \\
\hline Kadıköy - İSTANBUL / & ISTANBUL / TURKEY 34714 \\
\hline $\begin{array}{l}\text { e-posta: editor@rumelide. } \\
\text { tel: }+905057958124,+902167730\end{array}$ & $\begin{array}{l}\text { editor@rumelide.com, } \\
\text { +90 505 7958124, +90 } 216773 \text { o } 616\end{array}$ \\
\hline
\end{tabular}


Text analysis for translation and translation problems in a children's book example:Erich Kästner's Animal Meeting / Ü. Albiz (pp. 685-707)

\section{Fazit}

Ziel dieser Arbeit ist es, alle Einzelheiten der übersetzungsrelevanten Textanalyse von Nord in den Vordergrund $\mathrm{zu}$ stellen, insbesondere die textexternen und textinternen Faktoren und die Übersetzungsprobleme des Ausgangs- und Zieltextes. Dieses Ziel kann anhand von einem Kinderbuch auftreten. Die textexternen, textinternen Faktoren und die Wirkung des vorgenannten Kinderbuches feststellend, werden die Besonderheiten eines funktionalen Ansatzes dargestellt. Weil die Entscheidungen des Übersetzers oder der Übersetzerin sich auf die Übersetzungsprobleme auswirken, ist die Feststellung der Übersetzungsprobleme hinsichtlich der pragmatischen, konventionsbezogenen, sprachenpaarbezogenen, text(exemplar)spezifischen Übersetzungsprobleme von Bedeutung. Manche Übersetzungsprobleme insbesondere sprachenpaarbezogene Übersetzungsprobleme entstehen in diesem Kinderbuch. Aber die erfahrene Übersetzerin Süheyla Kaya beherrscht die Ausgangs- und Zielsprache ziemlich gut und anhand ihrer Übersetzungs- und Sprachkompetenz überwindet sie diese Übersetzungsprobleme. Und da dieses Werk ein Kinderbuch als Textart ist, ist der Empfängerkreis des Werkes mit den Kindern beschränkt. Deshalb übertragen sowohl der Ausgangstext als auch der Zieltext die gleiche Funktion. Man kann behaupten, dass die Textart dieses Werkes die Funktion des Ausgangstextes mit der Funktion des Zieltextes gleichsetzt und dafür die Übersetzerin eine erhebliche Rolle spielt.

\section{Literaturverzeichnis}

Cseho, Tamas (2007) Übersetzungsrelevante Analyse und kritische Übersetzung eines deutschen Zeitungsartikel, Germanistische Studien VI,63-80.

Kutlu, Serpil (2006) Übersetzungsprobleme in der Kinder und Jugendliteratur am Beispiel von Erich Kästers Roman "Das Fliegende Klassenzimmer" und der turkıschen Übersetzung "Uçan Sınıf", Hacettepe Universität, Instıtut für Sozialwissenschaften Abteilung für Deutsche Sprache und Literatur, Magisterarbeit, Ankara

Kautz, Ulrich (2002), Handbuch Didaktik des Übersetzens und Dolmetschens, Goethe Institut, 2. Auflage, München, Iudicum

Kästner, Erich (2000), Die Konferenz der Tiere, 8. Auflage Februar, Deutscher Taschenbuch Verlag GmbH \& Co. KG, München.

Kästner, Erich (2018), Hayvanlar Toplantısı, 17. Basım, Nisan, Can Çocuk Yayınları, Çev. Süheyla Kaya, İstanbul.

Nord, Christiana (1993), Einführung in das funktionelle Übersetzen, am Beispiel von Titeln und Überschriften, Francke Verlag, Tübingen und Basel.

Nord, Christiana (1999), Textanalyse: pragmatisch / funktional inkl. Handbuchtranslation, SnellHornby, Mary; Hönig, Hans ; Kußmaul Paul; Schmitt Peter A. (Hrsg.) , Zweite, verbesserteAuflage, StauffenburgVerlag, Tübingen.

Nord, Christiana (2009), Textanalyse und Übersetzen, Theoretische Grundlagen, Methode und didaktische Anwendung einer übersetzungsrelevanten Textanalyse,4., überarbeitete Auflage, Julius Groos Verlag Tübingen.

Nord, Christiana (2006) Loyalty and Fidelity in Specialized Translation, Artıgos E Comunıcações, Confluências - Revista De Tradução Cientifica E Técnica, N. ${ }^{\circ}$ 4, Maio 2006: 29-41.

Oittinen, Riitta (1999) inkl. HandbuchTranslation,Snell-Hornby, Mary; Hönig, HansG. ; Kußmaul Paul; Schmitt Peter A. (Hrsg.),, Zweite, verbesserteAuflage, StauffenburgVerlag, Tübingen.

Prunc, Eric (2002), Einführung in die Translationswissenschaft, Band1 Orientierungsrahmen,2. Erweiterte und verbesserte Auflage, Selbstverlag, Institut für Translationswissenschaft.

\begin{tabular}{r|l} 
Adres & Address \\
RumeliDE Dil ve Edebiyat Araştırmaları Dergisi & RumeliDE Journal of Language and Literature Studies \\
Osmanağa Mahallesi, Mürver Çiçeği Sokak, No:14/8 & Osmanağa Mahallesi, Mürver Çiçeği Sokak, No:14/8 \\
Kadıköy - İSTANBUL / TÜRKIYE 34714 & Kadıköy - ISTANBUL / TURKEY 34714 \\
e-posta: editor@rumelide.com & e-mail: editor@rumelide.com, \\
phone: +90 505 7958124, +90 2167730616
\end{tabular}


Reiss, Katharia (1993), Texttyp und Übersetzungsmethode, Der operative Text, 3.unveränderte Auflage, Julius Groos Verlag Heidelberg.

Stolze, Radegundis (2005), Übersetzungstheorien, Eine Einführung,4. Auflage, Günter Narr Verlag Tübingen.

https://www.europa-verlag.com/ber_uns.htm l,06.04.2021

https://www.dtv.de/verlag/ueber-uns-about-us/c-39, 06.04.202 